\title{
Macrolide resistance in Pseudomonas aeruginosa: implications for practice
}

\author{
James D. Chalmers \\ Affiliation: Division of Molecular and Clinical Medicine, University of Dundee, Dundee, UK. \\ Correspondence: J. D. Chalmers, Division of Molecular and Clinical Medicine, University of Dundee, \\ Ninewells Hospital and Medical School, Dundee, DD1 9SY, UK. E-mail: jchalmersवdundee.ac.uk
}

@ERSpublications

New research gives us reason for concern regarding long-term macrolide use http://ow.ly/g4Ap30b3lp3

Cite this article as: Chalmers JD. Macrolide resistance in Pseudomonas aeruginosa: implications for practice. Eur Respir J 2017; 49: 1700689 [https://doi.org/10.1183/13993003.00689-2017].

\begin{abstract}
Long-term low-dose macrolide use is rapidly becoming a standard treatment for a range of inflammatory lung diseases. In cystic fibrosis (CF), the 2014 annual report of the European Cystic Fibrosis Registry [1] shows that the use of macrolides is widespread, ranging from $10 \%$ of all CF patients in Austria, $38 \%$ in Spain, and $41 \%$ in the UK, to $44 \%$ in France, $48 \%$ in the Netherlands and $62 \%$ in Moldova. Macrolides are therefore one of the most frequently used therapies for CF in clinical practice [2]. In bronchiectasis not due to CF, data from the European Bronchiectasis Registry show that approximately $20 \%$ of patients are treated with macrolides, increasing to more than 30\% in specialist European centres [3-5]. In chronic obstructive pulmonary disease (COPD), long-term macrolide therapy is not yet widespread, but is likely to become more so with the release of the updated 2017 Global Strategy for the Diagnosis, Management and Prevention of COPD (GOLD) report that now recommends long-term low-dose macrolide therapy to prevent exacerbations in patients with GOLD type-D COPD and frequent exacerbations despite triple-inhaled bronchodilator and corticosteroid therapy [6].

The inflammation in CF, bronchiectasis and many patients with COPD is neutrophilic and macrolides represent one of the few treatment options that are able to combat neutrophilic inflammation [7]. Nevertheless, the mechanisms of macrolide benefit are hotly debated. Macrolides (azithromycin in particular) have been reported to reduce neutrophilic inflammation, reduce the production of pro-inflammatory cytokines, regulate mucus production, and inhibit the expression of virulence factors by bacteria including Pseudomonas aeruginosa [8-10]. A Cochrane review of long-term low-dose macrolide treatment in CF has shown improvements in lung function (approximately $4 \%$ in forced expiratory volume in $1 \mathrm{~s}\left(\mathrm{FEV}_{1}\right)$ ), a reduced frequency of exacerbations, and an increase in patient weight over 6 months of treatment (there was an absence of clear data beyond 6 months) [11, 12]. In bronchiectasis, 3 small but conclusive trials showed a reduction in exacerbations of around $50 \%$ with associated improvements in quality of life $[13,14]$.
\end{abstract}

Existing evidence suggests that macrolides may be most effective in patients with $P$. aeruginosa and this is where the bulk of evidence exists in CF [11-14]. In bronchiectasis, only the randomised BLESS trial (of erythromycin versus a placebo) examined possible response modifiers and showed a trend towards greater benefit in patients with baseline $P$. aeruginosa [14]. The apparently excellent response to macrolides of patients with $P$. aeruginosa has been one of the primary justifications for believing that the anti-inflammatory effects of macrolides are more important than their antimicrobial effects [15]. $P$. aeruginosa is considered intrinsically resistant to macrolides, demonstrating minimum inhibitory concentrations (MICs) of $>256 \mathrm{mg} \cdot \mathrm{L}^{-1}$ in standard growth media [16]. It uses multidrug-efflux systems to

Received: April 022017 | Accepted: April 032017

Conflict of interest: Disclosures can be found alongside this article at erj.ersjournals.com

Copyright CERS 2017 
efficiently remove macrolides from the cell [16] and hence guidelines may suggest the use of inhaled antimicrobials for P. aeruginosa infection in preference to macrolides, despite their greater ease of use [17]. However, in 2012, BuYcK et al. [16] demonstrated that macrolides may kill $P$. aeruginosa in vitro under physiological conditions similar to those in the airway. Increased permeability of the $P$. aeruginosa outer membrane is observed when exposed to serum, bronchoalveolar lavage (BAL) fluid or RPMI 1640 cell culture medium. Under these conditions, sufficient antibiotic may enter the cell to inhibit synthesis of outer membrane porins and efflux transporters, allowing macrolides to become effective antibacterials [16].

The realization that information from standard microbiological testing can be misleading is a familiar one in the field of chronic airway disease. It is well known that using MICs determined for serum is unhelpful when dealing with organisms in the lung, particularly when inhaled antimicrobials are used that achieve concentrations far above those in serum [18]. Populations of $P$. aeruginosa are heterogeneous such that different colonies may be resistant or sensitive to antibiotics [19] and, consequently, patients may respond to treatment with antibiotics that they are "resistant" to in vitro. To this we can now add the fact that macrolides (and potentially other antibiotics) may show entirely different sensitivity results depending on the growth media used.

Expanding on this previous work, this issue of the European Respiratory Journal features a manuscript by Mustafa et al. [20] wherein they investigate the potential for P. aeruginosa to develop resistance to macrolides in vivo. After all, it is logical that if macrolides have an antimicrobial effect against $P$. aeruginosa, the organism will be able to develop resistance. Mustafa et al. [20] used 333 clinical $P$. aeruginosa isolates obtained from patients with CF cared for at four centres in Belgium, France, Germany and the UK, respectively. They demonstrated the presence of macrolide-resistance in these "CF isolates" in eukaryotic growth media, which were not present in healthcare-associated pneumonia isolates where patients had not been exposed to chronic macrolide usage. Resistance mechanisms for macrolides, across multiple species of bacteria, are usually due to efflux pumps or modification of the ribosomal binding site of the antibiotic. In this study, MusTAFA et al. [20] demonstrated that efflux-pump inhibitors did not reverse macrolide resistance in the CF isolates. Furthermore, sequencing of the $23 \mathrm{~S}$ rRNA gene subsequently demonstrated six different mutations in the ribosomal target of the macrolides which, when transformed into a susceptible isolate, resulted in induced resistance to azithromycin. These data confirm that $P$. aeruginosa can acquire mutations in the $23 \mathrm{~S}$ rRNA gene rendering it nonsusceptible to macrolides. A direct relationship between chronic azithromycin use by patients and macrolide resistance in CF was observed that was statistically significant for the Liverpool epidemic strain [20]. This final data is not unexpected, but it is important in demonstrating that chronic antibiotic use is likely to drive mutations in $P$. aeruginosa in real life.

The clinical implications of this experimental work are important. It can no longer be assumed that macrolide effectiveness in $P$. aeruginosa is due to an anti-inflammatory mechanism and, if macrolide effects are partially or wholly due to antimicrobial activity, the potential for the development of resistance could mean a loss of macrolide effectiveness over time. Our ability to detect this has been limited to date, since the majority of CF studies are 6 months in duration and the longest studies in bronchiectasis and COPD are 12 months in duration [12-15]. Preliminary evidence from CF suggests that macrolides may indeed lose effectiveness with time. SAMson et al. [21] studied 68 patients who showed an initial reduction in exacerbations over the first 12 months of azithromycin treatment, followed by an increase in exacerbations back to baseline levels after 12 months. This is a problem that is not going to go away and which presents us with a key challenge for future work involving macrolides. The adult CF population in Europe is projected to rise by up to $75 \%$ by the year 2025 due to improved survival, while the prevalence of bronchiectasis not due to CF has increased by more than $30 \%$ in the past decade and is set to rise further [22-24]. These factors, along with the recent GOLD recommendations for COPD, mean that we are likely to see a continued rise in the use of long-term macrolide therapy. We need longer studies to determine if ongoing treatment with macrolides presents ongoing benefits, or if potential resistance results in loss of effectiveness. If the latter is true, patients could potentially discontinue therapy after 12 months with resulting benefits in terms of treatment burden and side-effects. Alongside this research priority, recent publications in the European Respiratory Journal regarding priorities in COPD and bronchiectasis have highlighted the need to identify which patients benefit most from macrolide treatment $[25,26]$. The potential for macrolides to cause resistance has to be taken seriously alongside other adverse effects such as drug-drug interactions, cardiovascular effects, hearing loss and the risk of inducing macrolide resistance in nontuberculous mycobacteria [27]. Recent evidence suggests that macrolide co-administration may antagonise the effect of inhaled tobramycin in CF, potentially through a different antibiotic resistance mechanism (the upregulation of efflux pumps as described by Nichols et al. [28]). This calls for careful selection of patients to receive macrolides. For example, existing data suggest that smokers respond poorly to macrolides in COPD; however, few other predictors have been identified [29, 30]. This should be 
addressed with new studies or secondary analyses of existing datasets. Macrolides are relatively unique among respiratory therapies and, while all therapies present a risk of side-effects to the individual, macrolides also present a threat to society by the spread of antimicrobial resistance. Further studies such as those by Mustafa et al. are needed if we are to understand the extent and clinical implications of the development of macrolide-induced resistance.

\section{References}

1 European Cystic Fibrosis Society Patient Registry (ECFSPR). Annual data report (year 2014). Karup, European Cystic Fibrosis Society, 2016. www.ecfs.eu/sites/default/files/general-content-files/working-groups/ ecfs-patient-registry/ECFSPR_Annual\%20Report\%202014_Nov2016.pdf. Date last accessed: April 212017.

2 Elborn JS, Bell SC, Madge SL, et al. Report of the European Respiratory Society/European Cystic Fibrosis Society task force on the care of adults with cystic fibrosis. Eur Respir J 2016; 47: 420-428.

3 Chalmers JD, Aliberti S, Polverino E, et al. The EMBARC European Bronchiectasis Registry: protocol for an international observational study. ERJ Open Res 2016; 2: 00081-2015.

4 McDonnell MJ, Aliberti S, Goeminne PC, et al. Multidimensional severity assessment in bronchiectasis: an analysis of seven European cohorts. Thorax 2016; 71: 1110-1118.

5 Aliberti S, Lonni S, Dore S, et al. Clinical phenotypes in adult patients with bronchiectasis. Eur Respir J 2016; 47: $1113-1122$.

6 Vogelmeier CF, Criner GJ, Martinez FJ, et al. Global Strategy for the Diagnosis, Management, and Prevention of Chronic Obstructive Lung Disease 2017 Report: GOLD Executive Summary. Eur Respir J 2017; 49: 1700214.

7 Gifford A, Chalmers JD. The role of neutrophils in cystic fibrosis. Curr Opin Haematol 2014; 21: 16-22.

8 Barr HL, Halliday N, Cámara M, et al. Pseudomonas aeruginosa quorum sensing molecules correlate with clinical status in cystic fibrosis. Eur Respir J 2015; 46: 1046-1054.

9 Schögler A, Kopf BS, Edwards MR, et al. Novel antiviral properties of azithromycin in cystic fibrosis airway epithelial cells. Eur Respir J 2015; 45: 428-439.

10 Tsai WC, Hershenson MB, Zhou Y, et al. Azithromycin increases survival and reduces lung inflammation in cystic fibrosis mice. Inflamm Res 2009; 58: 491-501.

11 Southern KW, Barker PM, Solis-Moya A, et al. Macrolide antibiotics for cystic fibrosis. Cochrane Database Syst Rev 2012; 11: CD002203.

12 Saiman L, Marshall BC, Mayer-Hamblett N, et al. Azithromycin in patients with cystic fibrosis chronically infected with Pseudomonas aeruginosa: a randomized controlled trial. JAMA 2003; 290: 1749-1756.

13 Chalmers JD, Aliberti S, Blasi F. Management of bronchiectasis in adults. Eur Respir J 2015; 45: 1446-1462.

14 Serisier DJ, Martin ML, McGuckin MA, et al. Effect of long-term, low-dose erythromycin on pulmonary exacerbations among patients with non-cystic fibrosis bronchiectasis: the BLESS randomized controlled trial. JAMA 2013; 309: 1260-1267.

15 Martinez FJ, Curtis JL, Albert R. Role of macrolide therapy in chronic obstructive pulmonary disease. Int J Chron Obstruct Pulmon Dis 2008; 3: 331-350.

16 Buyck JM, Plesiat P, Traore H, et al. Increased susceptibility of Pseudomonas aeruginosa to macrolides and ketolides in eukaryotic cell culture media and biological fluids due to decreased expression of oprM and increased outer-membrane permeability. Clin Infect Dis 2012; 55: 534-542.

17 Pasteur MC, Bilton D, Hill AT. British Thoracic Society guideline for non-CF bronchiectasis. Thorax 2010; 65: Suppl. 1, i1-i58.

18 Konstan MW, Flume PA, Galeva I, et al. One-year safety and efficacy of tobramycin powder for inhalation in patients with cystic fibrosis. Pediatr Pulmonol 2016; 51: 372-378.

19 Gillham MI, Sundaram S, Laughton CR, et al. Variable antibiotic susceptibility in populations of Pseudomonas aeruginosa infecting patients with bronchiectasis. J Antimicrob Chemother 2009; 63: 728-732.

20 Mustafa M-H, Khandekar S, Tunney MM, et al. Acquired resistance to macrolides in Pseudomonas aeruginosa from cystic fibrosis patients. Eur Respir J 2017; 49: 1601847.

21 Samson C, Tamalet A, Thien HV, et al. Long-term effects of azithromycin in patients with cystic fibrosis. Respir Med 2016; 117: 1-6.

22 Burgel PR, Bellis G, Olesen HV, et al. Future trends in cystic fibrosis demography in 34 European countries. Eur Respir J 2015; 46: 133-141.

23 Quint JK, Millett ER, Joshi M, et al. Changes in the incidence, prevalence and mortality of bronchiectasis in the UK from 2004 to 2013: a population-based cohort study. Eur Respir J 2016; 47: 186-193.

24 Ringshausen FC, de Roux A, Diel R, et al. Bronchiectasis in Germany: a population-based estimation of disease prevalence. Eur Respir J 2015; 46: 1805-1807.

25 Aliberti S, Masefield S, Polverino E, et al. Research priorities in bronchiectasis: a consensus statement from the EMBARC Clinical Research Collaboration. Eur Respir J 2016; 48: 632-647.

26 Celli BR, Decramer M, Wedzicha JA, et al. An official American Thoracic Society/European Respiratory Society statement: research questions in COPD. Eur Respir J 2015; 45: 879-905.

27 Albrecht C, Ringshausen F, Ott S, et al. Should all adult cystic fibrosis patients with repeated nontuberculous mycobacteria cultures receive specific treatment? A 10-year case-control study. Eur Respir J 2016; 47: 1575-1577.

28 Nichols DP, Happoldt CL, Bratcher PE, et al. Impact of azithromycin on the clinical and antimicrobial effectiveness of tobramycin in the treatment of cystic fibrosis. J Cyst Fibros 2017; 16: 358-366.

29 Agusti A, Bel E, Thomas M, et al. Treatable traits: toward precision medicine of chronic airway diseases. Eur Respir J 2016; 47: 410-419.

30 Han MK, Tayob N, Murray S, et al. Predictors of chronic obstructive pulmonary disease exacerbation reduction in response to daily azithromycin therapy. Am J Respir Crit Care Med 2014; 189: 1503-1508. 